\title{
DESAFÍOS Y PERSPECTIVAS EN LA INVESTIGACIÓN SOBRE EL MAGISTERIO
}

\author{
Sigfredo Chiroque Chunga*
}

\begin{abstract}
RESUMEN: En investigación en general y en investigación educativa y sobre el magisterio importa precisar el "para qué" y el "para quién". Ambas decisiones se deberían conjugar en la opción de transformar la sociedad. Esto es posible hacerlo en nuestros países, a pesar del rasgo de desigualdad y de relativo control existente en el desarrollo investigativo. En investigación magisterial, deberíamos superar el control económico-financiero, de enfoque metodológico y el que, a veces, se esconde bajo el rótulo de hacer "incidencia política". En América Latina, hemos ido precisando la temática de investigación sobre el magisterio. Pero, su enfoque ha tenido que ver, por un lado, con los postulados neoliberales y de la investigación cualitativa-etnográfica y, por otro lado de la educación liberadora y de las prácticas sociales del magisterio latinoamericano. Desde esta última opción surgen importantes perspectivas que hay necesidad de precisar y concertar.
\end{abstract}

Palabras clave: Magisterio. Educación. América Latina.

\section{Challenges AND PROSPECTS IN THE RESEARCH ON TEACHING}

ABSTRACT: In research in general and in research on education and teaching it is worth specifying "what for" and "who for". Both decisions should combine in the option of transforming society. This can be done in our countries, in spite of the inequality divide and of the relative control on the development of research. In research on teaching, we should overcome the economical-financial control, with its methodological focus, and the one that - sometimes - hides under the name of making "political incidence". In Latin America, we have specified the themes of research on teaching. Nevertheless, their fo-

Sociólogo, profesor e investigador del Instituto de Pedagogía Popular (IPP) de Lima, Perú. E-mail: schiroque@ipp-peru.com

Educ. Soc., Campinas, vol. 28, n. 99, p. 483-498, maio/ago. 2007 
cuses have to do with the assumptions of neo-liberalism and qualitative-ethnographic research, on the one hand, and with those of the liberating education and of the social practices of Latin-American teaching, on the other. Important prospects emerge from this last option, which need to be specified and coordinated.

Key words: Teaching. Education. Latin America.

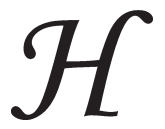

ay una paradoja en el desarrollo de la investigación científica en general. Por un lado - por razones de una creciente competencia entre empresas transnacionales ${ }^{1}-$, ella se ha desarrollado de manera intensiva en las naciones ricas; mientras tiene débil desarrollo en países pobres, como los de América Latina. Dentro de esta debilidad, el campo específico de la investigación educacional y sobre el magisterio resulta aún más reducido. Esta situación no podemos olvidarla al momento de intentar precisar los desafíos y perspectivas de la investigación sobre magisterio.

Es indudable que el análisis de toda realidad presente o futura se ve teñida por la intencionalidad o enfoque que se persigue. En este sentido, nos interesa explicitar inicialmente la intencionalidad que le estamos asignando a la práctica investigativa, desde una opción crítica y de educación liberadora.

\section{Sentido de un debate}

Es lugar común admitir la importancia de la producción sistemática de conocimiento sobre realidades de la naturaleza y de la sociedad, con fines descriptivos, explicativos o de transformación y predicción. Sin embargo, en la medida que ha ido avanzando el modelo neoliberal, hasta el conocimiento producido por la práctica investigativa se ha ido transformando en mercancía. Y claro está, la producción, distribución y usoconsumo de esa mercancía han ido asumiendo los rasgos de una economía de mercado. Los desafíos y perspectivas de la investigación educacional y sobre el magisterio se ven teñidas necesariamente por este marco del sistema.

Siendo así, importa plantear una opción preliminar, en función a la misma teleología de nuestra práctica social e investigativa: o ella se pone al servicio de la resistencia a la actual hegemonía y construcción 
de una nueva hegemonía; o ella simplemente sirve a la manutención del hegemónico sistema injusto de sociedad. Esto tiene que ver con la determinación de la finalidad y de los receptores de la práctica investigativa.

La opción del para qué investigar se confunde con el para quién investigar. Y tomar una decisión - en ambos casos - conlleva derivaciones en posibilidades financieras para realizar los estudios y aún opciones referidas a los paradigmas de investigación a ser usados. El enfoque de la investigación resulta de importancia no solamente teórica, sino práctica, como lo reconocía Coraggio (1996) hace 10 años.

En el presente documento, nos reafirmamos en una investigación al servicio de la transformación de la educación y de la sociedad. Y en este sentido, orientada hacia personas e instituciones que pugnan por el cambio. Siendo así, desde un enfoque liberador, podemos preguntarnos: ¿Cuál es el contexto de la investigación educativa y sobre el magisterio dentro de un marco de globalización neoliberal?, ¿Cuáles son los desafíos y las perspectivas de la investigación socio-educacional y de la investigación sobre el magisterio en este contexto?, ¿Las instituciones y personas que realizamos investigación en este campo tendremos la suficiente autonomía para producir y distribuir conocimiento con relativa autonomía, según nuestras propias opciones ajenas a los intereses del capital?

Contexto de la investigación educativa y sobre magisterio

La investigación educativa en general y la referida al magisterio en particular se ubican dentro de un contexto de desarrollo desigual en la producción y distribución de Ciencia-Tecnología-Innovación (Сті), así como en un marco de control estructural en su enfoque, temática y resultados.

\section{Desarrollo desigual}

El desarrollo de СтI es parte de la agenda internacional. En foros y declaraciones internacionales, ${ }^{2}$ se proclama con cierto lirismo de "compartir el conocimiento". Pero esto objetivamente no se da.

Con datos 1998 - que seguramente no han tenido mayores modificaciones - América Latina en su conjunto estaba a la zaga en cuanto a inversión en Ciencia y Tecnología (Investigación + Desarrollo = I 
+ D). Ésta apenas llegaba al 1.7\% del total, siendo Brasil, México, Argentina y Colombia los que más destacaban.

No queremos abundar en esta situación ya conocida. Solamente queremos destacar que el desarrollo de CTI en los países ricos tiene que ver con las necesidades de la industria, principalmente bélica. En la medida que el conocimiento científico y tecnológico se ha convertido en una fuerza de producción, entonces, reproduce, de alguna manera, la estructura social. Se convierte, así, en herramienta necesaria del neoimperialismo:

La ciencia moderna es inseparable de la política porque, en última instancia, es un instrumento de poder y porque más recientemente se ha convertido en uno de los ejes sobre los que se transforma la estructura social (...).

En los hechos, el surgimiento de la política cientifica contemporánea está directamente vinculado con la guerra. El documento al que se considera como acta fundacional de la política cientifica ("Science, the Endless Frontier" de Vannevar Bush) fue escrito en respuesta a un requerimiento del presidente Roosevelt quien deseaba saber cómo podían los Estados Unidos valerse de la ciencia para ganar las batallas (...). (Albornoz, 2001, p. 3)

El crecimiento de las empresas transnacionales se correlaciona con el intensivo desarrollo de CTI en los países donde están sus matrices centrales. Este desarrollo resulta absolutamente desigual comparado al existente en nuestros pueblos.

\section{Control}

Explicando mejor esta asimetría importa señalar que: cuanto más cercanos a los intereses del capital son los conocimientos que devienen de la investigación, mayor control existirá en su producción, temática, enfoque y en la distribución-uso de los mismos.

Las investigaciones de carácter productivo y/o tecnológico tienden a un control más riguroso por el sistema, que está dispuesto a invertir más en este campo. Mientras que las investigaciones socio-educativas - en la medida que no tengan un nexo muy directo - con los intereses del sistema no solamente tendrán menos recursos de inversión, sino que se reducen los mecanismos de control para su producción, enfoque y distribución-uso de sus resultados. 
Lo que acabamos de decir explica, de alguna manera, el relativo desarrollo de las investigaciones sociales en nuestros pueblos y - dentro de ellas - el desarrollo de la investigación educativa, desde la tercera década del siglo XX, principalmente en países como Colombia, México, Brasil y Argentina (Narodowski, 1999). Es decir, aparentemente la investigación educativa se habría desarrollado con cierta independencia, siguiendo una suerte de "tradición a la autonomía académica” (Miñana Blasco, 2002, p. 3).

Analizando el desarrollo de la investigación socio-educativa en América Latina para las década de 1970 y 1980, Juan Carlos Tedesco encontraba un alta correlación entre modelos sociales vigentes o que se colocaban como alternativos y enfoques educativos para la cual se hace investigación. Tedesco reconocía: el paradigma de la teoría educativa liberal, el paradigma economicista (capital humano, recursos humanos) y el paradigma de los enfoques crítico-reproductivistas. Todos ellos, según Tedesco (1986), guardaban relación a momentos históricos, explicados por las dinámicas sociales.

El relativo desarrollo de la investigación no es casual. De manera directa o indirecta se genera un verdadero control. Destacamos tres manifestaciones: el control económico-financiero, el control metodológico y el control con enfoque de incidencia política.

\section{Control económico-financiero}

No es novedad, las dificultades existentes para encontrar financiamiento para la realización de investigación. Son escasos los gobiernos de América Latina que derivan recursos para la investigación; pero también existe dificultad en la cooperación internacional para la ejecución de estudios, principalmente de aquellos con enfoque social y mucho menos cuando asumen un enfoque de transformación. Como que el mismo sistema delimitase la creación autónoma y sistemática de conocimiento, generando una suerte de sutil control financiero.

En la medida que las investigaciones educativas han servido a propósitos de cambio, las instancias formales que apoyan la producción, distribución y uso de conocimiento científico-tecnológico han relativizado su apoyo. 


\section{Control de enfoque metodológico}

La misma inteligencia que postula el status quo ha encontrado mecanismos de control de las investigaciones orientadas hacia el cambio. Queremos destacar dos de primera importancia: una referida al enfoque metodológico de las investigaciones y otra en referencia a la definición del "para quién" se hace investigación.

Pierre Bourdieu encuentra que esto se da cuando se busca separar la investigación de la teoría, postulándose el método al margen del enfoque. Siendo así, las investigaciones se reducen al mero uso de técnicas empíricas y de medición; o a los simples postulados deseables. La investigación se postula: o a partir de simples situaciones que "son" o a partir de enunciados doctrinarios de lo que "debe ser". Se oscila entre el empirismo y el idealismo de nuevo cuño.

Hoy el mayor peligro que enfrentamos es la creciente separación entre la teoría y la investigación que vemos por todas partes y que alimenta el concomitante crecimiento de la perversión metodológica y la especulación teórica (...). La investigación positivista y la teoría tecnicista van de la mano, se complementan y se halagan mutuamente. (Bourdieu y Wacquant, 2005, p. 249-250)

El control referido al enfoque metodológico repercute en la calidad de los resultados investigativos. Ellos se presentan como "data" que sirve casi exclusivamente para el mejoramiento del sistema o como "postulados doctrinarios", descontextualizados de las dinámicas sociales y políticas.

Hace unas décadas, era normal que personas e instituciones progresistas desarrollasen su práctica social o investigativa pensando en que el movimiento popular acumulase directamente los resultados de su práctica. Éste movimiento popular, con el apoyo recibido, potenciaba y mejoraba su quehacer político, buscando alterar el sistema.

\section{Control con enfoque de "incidencia política"}

En los últimos tiempos, se está poniendo de moda la tesis de la "incidencia política”, entendida como una práctica deliberada para influir en aquellos que toman decisiones sobre políticas, preferentemente 
desde alguna instancia del Estado (Sprechmann y Pelton, 2001). Siguiendo esta tesis - hasta cierto punto falaz - la práctica social de algunas personas e instituciones replantea su direccionalidad. Sus resultados se orientan en lo fundamental hacia alguna instancia del Estado.

La incidencia en la práctica social no tendría nada de malo si ella se asume como generalización de resultados. La limitación se encuentra en que los resultados son cooptados por el sistema y esto sirve como prerrequisito para derivación de recursos. Es decir, el mismo financiamiento de las investigaciones (y otras prácticas sociales) queda supeditado a la "incidencia política" y ésta adquiere una orientación de insumo para la reproducción del sistema.

\section{La falacia de la incidencia política}

Con esta orientación y en el caso de las investigaciones, los sectores populares que pugnan por el cambio ya no son receptores inmediatos y privilegiados de los nuevos conocimientos producidos, sino que estos resultados son directamente cooptados por personas que toman decisiones en diversas instancias del sistema. Estas personas e instancias del sistema, generalmente y por función, buscan su reproducción.

Un enfoque dicotómico de los dos caminos señalados puede resultar perjudicial e ingenuo; en la medida que pierde de vista lo central: que nuestra práctica investigativa se ponga al servicio de la resistencia a la actual hegemonía y construcción de una nueva hegemonía. Evidentemente esta opción debe priorizar a los grupos e instancias de cambio, que pueden estar dentro de Estado, ${ }^{3}$ pero que básicamente se encuentran fuera de él.

Desde la intelectualidad progresista, se han generado mecanismos de resistencia al control. Se han realizado importantes avances, principalmente en lo que se refiere al enfoque con el cual se bordan los problemas (Akkari y Perez, 1998); pero también se han creado redes y espacios comunes de reflexión. Las nuevas tecnologías de información se vienen utilizando con mucha eficacia para el intercambio de información y para la producción-distribución de nuevo conocimiento con enfoque de cambio. Esta tendencia promisoria debe ser retomada y ampliada, también la temática de la investigación sobre los docentes en América Latina y en los países del Norte. 


\section{La investigación sobre el magisterio}

Queremos colocar algunas pistas sobre investigación sobre el magisterio. Preferimos hablar de "investigación sobre el magisterio" y no sobre "trabajo docente", pues la primera categoría no sólo pone énfasis en el sujeto colectivo, sino que permite un abordaje del mismo que va más allá de su práctica laboral. El trinomio trabajador-trabajo-producto se percibe mejor cuando usamos la categoría "magisterio" o "docentes", evitando yuxtaposiciones o fragmentaciones funcionales, que hacen perder la visión de totalidad y del sujeto con sus prácticas y relaciones - históricamente dadas - como centro de todo análisis. No queremos caer en una visión meramente funcional de los docentes. Como dice Giroux (1997, p. 173):

Una de las amenazas más importantes a que tienen que hacer frente los futuros y los actuales profesores de la escuela pública es el creciente desarrollo de ideologías instrumentales que acentúan el enfoque tecnocrático tanto de la formación del profesorado como de la pedagogía del aula.

\section{Desarrollo histórico en América Latina: de objeto a sujeto de estudio}

Es importante advertir que la relevancia del magisterio - como tema de la investigación educacional - guarda relación con los paradigmas educativos. Cuando el eje educacional se le ubica en la enseñanza, también se prioriza al que enseña. Cuando dicho eje es el aprendizaje, se prioriza al que aprende. Cuando asumimos que el eje es la interacción de los sujetos que aprenden y enseñan, esta relación adquiere importancia en la investigación.

Hasta la década de 1970, en América Latina, predominaba el paradigma de la enseñanza. Las investigaciones educativas se centraban en estos procesos y también en el rol enseñante del magisterio. Su labor didáctica era centro de estudios.

En las década de 1980 y 1990, el énfasis que se dio al aprendizaje derivó en que las investigaciones educativas se reorientasen hacia el sujeto que aprende y sus condiciones. Los temas de calidad y equidad en los aprendizajes, el rendimiento escolar, la eficiencia, la educabilidad y gestión escolar se pusieron a la orden del día.

Creemos que la temática magisterial recupera peculiar relevancia en la investigación de América Latina en los 20 últimos años. Esta 
relevancia podemos atribuirla a la confluencia, por un lado, de factores derivados de los postulados neoliberales y de la investigación cualitativaetnográfica y, por otro, de la educación liberadora y de las prácticas sociales del magisterio latinoamericano. Veamos rápidamente cada uno de ellas.

- Docente como factor a quien debe atribuirse la crisis de la educación - El modelo neoliberal busca "chivos expiatorios" a quien atribuir la crisis de la educación en nuestros países. Lejos de centrar la explicación de esta crisis en las condiciones de educabilidad (factores exógenos al sistema), señala al maestro y maestra como la principal determinación de la pobreza educativa. En este marco, se realizan estudios para demostrar la reducida calificación de los maestros o su nexo con los bajos rendimientos estudiantiles.

- Investigación cualitativa y etnográfica - Desde una perspectiva de estudios cualitativos y de etnografía educativa, se recupera en los 20 últimos años la investigación de los sujetos de la educación y en ella se levanta la investigación del "maestro como trabajador", como uno de los temas más estudiados (Miñana Blasco, 2002, Ob. c., p. 28).

- La educación liberadora - Un enfoque meramente reproductivista de la educación relativizaba su función liberadora y, por lo tanto, la investigación educacional. Paulatinamente fuimos tomando conciencia que la educación no es una categoría abstracta, sino práctica social e histórica y, por lo tanto, de práctica que se origina y se orienta hacia sujetos históricamente determinados. Los diversos sujetos de la educación - llámense maestros o comunidad - son finalmente quienes le hacen teñir de intereses y de ideología. Por ello, la educación, puede adquirir orientaciones contradictorias. Como dice Chomsky (2006, p. 2):

Piensa en una herramienta: un martillo. Al martillo no le interesa si es utilizado para romperle el cráneo a alguien. Lo puedes utilizar con diferentes propósitos. Del mismo modo la educación puede servir para controlar y subordinar a las personas e imponerles doctrinas que las marginan; o puede ser utilizada para que las personas se liberen. Tomemos como ejemplo el logro más extraordinario de la historia para crear una sociedad libre: la revolución anarquista de España en 1936. Ésta no fue una revolución espontánea, sino, más bien, un acto premeditado que tomó décadas en consolidarse. 
Trabajo, organización y programas especiales de educación construyeron en las mentes de los ciudadanos la idea de una sociedad libre. Cuando la oportunidad apareció, la aprovecharon. La educación puede servir para ese fin, pero también puede servir para disciplinar y controlar.

- Compromiso del magisterio en el cambio social - Desde un enfoque popular, las tesis mecanicistas se han ido superando y la práctica educativa y de los maestros fue recuperando su verdadero sentido de inserción en la lucha de clases y, específicamente, en su aporte para la resistencia contra la hegemonía vigente y la construcción de una nueva hegemonía. Paulo Freire ha sido uno de los pensadores que más ha influido en la recuperación del rasgo liberador de la educación y más específicamente del rol del magisterio en la transformación de la sociedad y de la educación (Freire, 1997). ${ }^{4}$

En varios países de nuestra América y del mundo, los docentes se han colocado como un contingente del movimiento popular y esto ha devenido en que se debe reformular el mismo pensamiento que se tiene sobre los docentes (Kincheloe, 2001).

Sin embargo, la reorientación del magisterio latinoamericano todavía se encuentra en construcción. No siempre los cambios educativos en América Latina han tenido la comprensión de los docentes y de sus organizaciones. La lógica neoliberal como que se ha dado la mano, en algunos casos, con la lógica mecánica y economicista de los gremios magisteriales, como lo advierte Torres (2000). Cuesta a los docentes - y principalmente a sus organizaciones gremiales - pensar que ellos, como sujetos, y los estudiantes también como sujetos, tienen derechos. El desarrollo de unos no puede darse al margen, aplastando o en dicotomía al derecho de los otros, como lo quieren el neoliberalismo y algunos gremios docentes. Importa que los derechos de los sujetos que enseñan se desarrollen en concordancia con los derechos de los sujetos que aprenden y viceversa (Chiroque Chunga, 2004).

La investigación sobre el magisterio generalmente se hizo con una orientación positivista, en la medida que se asumía que la reforma educativa era una simple cuestión técnica y, en este marco, los maestros eran simples operadores de esta transformación tecnocrática. Sin embargo al explicitarse mejor el carácter social y político de los cambios educativos -, también los estudios sobre los docentes se comenzó a encararlos 
con esta óptica política, donde importa relacionar la práctica docente con la correlación de fuerzas y la hegemonía de poder existente en nuestros pueblos, como lo señala Connell (2004).

La investigación sobre el magisterio generalmente ha venido avanzando asumiendo diversos rasgos de los docentes como objetos de estudio, donde los maestros y maestras son simple fuente de información. Sin embargo, en los últimos tiempos como que se abre una nueva perspectiva al considerarse que los maestros son productores de saber pedagógico y que éste debe ser democratizado. Siendo así, el saber producido por los docentes debe ser "objetivado, sistematizado, publicado, debatido-conversado, criticado-deconstruido y reconstruido". Y en todas estas tareas, el maestro pasa a tener un rol protagónico: de objeto de investigación pasa a ser sujeto de investigación sobre su propia práctica docente. ${ }^{5}$

\section{Construyendo perspectivas}

Asumo que las "tendencias" ponen el énfasis en la continuidad de las situaciones; mientras que las "perspectivas" - si bien recogen las tendencias - dar margen para la ruptura y la discontinuidad. Por ello, asumo las perspectivas como construcción crítica de un proceso, por parte de un sujeto colectivo. Y en este marco y considerando todo lo que hemos dicho, quisiera aportar con algunas sugerencias para la construcción colectiva de las perspectivas de la investigación sobre magisterio, con enfoque de cambio.

Para organizar nuestras sugerencias sobre la investigación aplicada al magisterio, queremos utilizar un enfoque sistemático, sin que ello nos confunda con los postulados del denominado "pensamiento sistémico", que formaliza el pensar la realidad como algo invariante (Roitman Rosenmann, 2004).

(1) Investigación magisterial que responda a la situación realmente existente en nuestros paises, pero también en función situaciones deseables y posibles - Importa estudiar el tipo de maestro que requerimos para responder a los rasgos socioculturales realmente existentes en nuestros pueblos, pero también a los proyectos de país que tenemos. 
En el campo educacional, requerimos un maestro no solamente para responder a situaciones educativas actuales sino a un Proyecto Educativo Nacional. Bien sabemos que, a nivel micro, encontramos un currículo oculto; pero, de igual manera y a nivel macro, encontramos para cada uno de nuestros países "Proyectos Educativos" ocultos, que nada tienen de nacionales. Ellos corresponden al sistema y al neoliberalismo transnacional. Por ello, importa explicitar estos Proyectos y en función a ellos elaborar investigaciones sobre el magisterio;

\section{Componentes sistemáticos para análisis de situación y perspectivas de la investigación educacional y magisterial}

(2) Investigación magisterial asumiendo no solamente la existencia material y objetiva de los docentes, sino también las representaciones producidas sobre ellos - Importa estudiar no solamente la objetividad de los maestros, sino también su dimensión subjetiva, así como la subjetividad que sobre él tiene la sociedad. Al respecto, dice Tenti Fanfani (2003, p. 77):

El magisterio, como todo grupo o clase social, tiene una existencia doble. El estamento magisterial tiene un primer nivel de existencia que podemos denominar objetivo-material. Desde este punto de vista posee una serie de propiedades que pueden medirse a través de la estadística social: edad promedio, ingres, composición sexual, escolaridad, lugar de residencia, propiedad etc. Pero este nivel no agota toda la realidad de un fenómeno social. Éste existe también como representación y como reconocimiento socialmente producido.

(3) Investigación magisterial que conjugue y guarde coherencia entre la opción teórica y la opción metodológica - $\mathrm{Ni}$ estudios que se queden en la simple "data" empírica desagregada, ni otros que se queden en postulados abstractos, yuxtapuestos y ahistóricos. Importa abordar el tema maestro como una totalidad integrada a otras totalidades. El estudio de alguna dimensión magisterial tiene que hacerse con este criterio:

Las flaquezas de la teoría social no se originan (...) en la 'incapacidad' de alcanzar una 'generalidad presuposicional' y una 'multidimensionalidad', sino en una división social del trabajo científico que divide, deifica y 
compartimenta los momentos de construcción del objeto sociológico en especialidades separadas. Premiando de esa manera la 'audacia sin rigor' de la filosofía social y el 'rigor sin imaginación' del positivismos hiperempirista. (Wacquant, 2005, p. 64-65)

(4) Investigación magisterial que precise el para qué y para quiénes elaborar el nuevo conocimiento - Aquí importa establecer con claridad el nexo de los resultados de las investigaciones con el movimiento social y popular, así como con núcleos del aparato estatal. No podemos supeditar la investigación al criterio de la denominada "incidencia política", pero tampoco rechazar una necesaria influencia en las políticas sociales. Desde nuestra perspectiva, cabe hacer esta incidencia fundamentalmente con y desde los grupos sociales organizados de la población.

(5) Matriz de temas y concertación de esfuerzos - Sería importante establecer un conjunto de temas-problemas de investigación sobre magisterio, a nivel de cada país y de la región. Para definir temas de investigación, puedo optar por 4 caminos:

- Hacer un análisis de los componentes implícitos en "desarrollo integral del magisterio";

- Explicitar lo que estudia y no estudia la comunidad científica sobre el magisterio;

- Establecer los temas relacionados a los problemas de los sistemas educativos en referencia al magisterio ("lo que es"); y/o

- Establecer una utopía del sector: "lo que debe ser".

El primer camino me ayuda como un simple trabajo lógico y pensante. El segundo camino me sirve de antecedente; mientras el estudio de simples problemas existentes puede llevarme sólo a mejorar el sistema existente. El cuarto camino me lleva a estudiar lo deseableposible y puede sesgarme a una suerte de idealismo. Importa asumir los 4 enfoques como complementarios.

La concertación de estos temas-problemas de investigación - a nivel nacional y regional - nos permitiría racionalizar recursos escasos; mejorar en calidad investigativa; aportar en creación de Ciencia-TecnologíaInnovación (CTI); generar condiciones para superar la dicotomía entre 
Desafíos y perspectivas en la investigación sobre el magisterio

teoría y metodología; intercambiar formas eficaces en la distribución de los resultados de la investigación; y en el uso adecuados de los mismos, en una perspectiva liberadora.

(6) Mejoramiento en la determinación y manejo de procesos y de recursos para la investigación sobre magisterio - La experiencia de la REDESTRADO debe ampliarse y profundizarse. Esto nos permitiría aunar esfuerzos para calificar a los investigadores presentes y futuros, mejorar en la captación y uso de los recursos financieros; democratizar fuentes de información y documentación; así como promover de manera concertada el desarrollo de cultura investigativa en la comunidad educativa y población magisterial. Los maestros y maestras deben recuperar su condición de productores de conocimiento, orientando sus resultados a la transformación de la educación de nuestros pueblos y de la misma sociedad.

Recebido em maio de 2007 e aprovado em junho de 2007.

\section{Notas}

1. Decimos "competencia formal" puesto que, en la globalización en marcha, la transnacionalización del capital conlleva en términos reales el control de los capitales nacionales. La competencia, entonces, no es entre países sino entre grandes empresas transnacionales.

2. Ver, por ejemplo, Informe anual 1998/1999 del Banco Mundial sobre el conocimiento; o Conferencia Mundial de la Ciencia, convocada por la UnesCo (2001).

3. Con esta observación, quiero rescatar a personas e instancias que pugnan por el cambio, también desde el aparato estatal. Esto se da con mayor claridad, en casos como Brasil, Venezuela y Bolivia. Y claro está, en situaciones donde el Estado está al servicio de los intereses populares, como es Cuba.

4. En esta obra, Freire analiza, desde diversas entradas, el papel de compromiso por el cambio que deben tener los maestros y maestras.

5. Existe abundante información sobre este enfoque. Destacamos dos documentos: Suárez (2005) e Rodríguez y Ramos (2006).

\section{Referencias bibliográficas}

ALBORNOZ, Mario. Política científica y tecnológica. Una visión desde América Latina. Revista Ibeoroamericana de Ciencia, Tecnología, Sociedad e Innovación, Madrid, n. 1, p. 1-9, sept.-dic. 2001. 
CORAGgIO, J. L. Sobre la investigación y su relación con los paradigmas educativos. Cuadernos de Educación, Buenos Aires, n. 3, p. 77-89, sept. 2000.

NARODOWSKI, M. La investigación educativa en América Latina: una respuesta a Akkari y Pérez. Education Policy Análisis Archives, Phoenix, v. 7, n. 2, p. 1-3, ene. 1999.

MIÑANA BLASCO, C. Producción y circulación de conocimiento en el campo de la politica educativa y la gestión en América Latina. Santafé de Bogotá: Universidad Nacional de Colombia; Programa RED, 2002.

TEDESCO, J.C. Los paradigmas de la investigación educativa. Revista Colombiana de Educación, Santafé de Bogotá, n. 18, 1986.

BOURDIEU, P.; WACQUANT, L. Una invitación a la sociología reflexiva. Buenos Aires: Siglo XXI, 2005. p. 249 y 250.

SPRECHMANN, S.; PELTON, E. Guias y herramientas para la incidencia política. Atlanta: CARE, 2001.

AKKARI, A.; PÉREZ, S. Investigación educativa en América Latina. Education Policy Análisis Archives, Arizona State University, v. 6, n. 7, p. 1-5, 1998.

GIROUX, H.A. Los profesores como intelectuales. Hacia una pedagogía crítica del aprendizaje. Barcelona: Paidós, 1997. p. 173.

CHOMSKY, N. Una sociedad decente eliminaría el rol del líder. El Comercio, Lima, Entrevista, p. 2, 29 oct. 2006.

FREIRE, P. Pedagogía de la autonomía. Madrid: Siglo XXI, 1997.

KINCHELOE, J.L. Hacia una revisión crítica del pensamiento docente. Barcelona: Octaedro, 2001.

TORRES, R.M. Reformadores y docentes. El cambio educativo atrapado entre dos lógicas. En: Los docentes, protagonistas del cambio educativo. Santafé de Bogotá: SECAB, 2000.

CHIROQUE CHUNGA, S. 1998-2003. Conflicto en el sistema educativo peruano. En: Estudio de los conflictos en los sistemas educativos de América Latina. Santiago de Chile: OREALC; UNESCO, 2004. 
CONNEL, R.W. Pobreza y educación. Crítica al neoliberalismo en educación. En: Gentili, P. (Coord.). Pedagogía de la exclusión. México: Universidad Autónoma de la Ciudad de México, 2004.

SUÁREZ, D. Los docentes, la producción del saber pedagógico y la democratización de la escuela. En: Anderson, G. y otros. Escuela. Producción y democratización del conocimiento. Buenos Aires: Gobierno de Buenos Aires; unesco, 2005.

RODRÍGUEZ, A.; RAMOS, M.T. Narrativa docente, práctica escolar y reconstrucción de la memoria pedagógica. En: CAEPLla, J. y otros. Criterios y métodos de una educación popular y liberadora. Lima: Instituto de Pedagogía Popular, 2006. (En prensa).

ROITMAN ROSENMANN, M. El pensamiento sistémico. Los orígenes del social-conformismo. México: Siglo XXI, 2004.

TENTI FANFANI, E. El oficio del maestro: contradicciones iniciales. En: Alliaud, A.; Duschatzky, L. (Comp.). Maestros. Formación, práctica y transformación escolar. Buenos Aires: Niño y Dávila, 2003. p. 77.

WACQUANT, L. Hacia una praxeología social: la estructura y la lógica de la sociología de Bourdieau. En: Bourdieau, P.; WaCQUANT, L. Una invitación a la sociología reflexiva. Buenos Aires: Siglo XXI, 2005. p. 64-65. 